\title{
Peak Streamflow Determinations in Nevada: A Cooperative Program with the USGS and Nevada Department of Transportation
}

\section{Background}

Floods are one of the most costly and frequent natural disasters in Nevada. For example, the 1997 New Year's flood has been estimated to have caused more than $\$ 1$ billion in damage across northern Nevada (Truckee River Flood Management Authority, 2017). In 2014, more than
2 miles of Interstate 15 in southern Nevada was heavily damaged by the remnants of Hurricane Norbert combined with monsoonal rains (Sutko, 2015). Flooding in Nevada is highly variable in cause and the season of the year. Flooding can be caused by snowmelt, rain on snow (fig. 1), and flash flooding during thunderstorms. Peak streamflow estimates are critical for planning by government agencies; designation of flood zones; and design of infrastructure including culverts, bridges, and roadways. In order to provide accurate estimates of flood frequencies, long-term data collection of peak streamflows would be needed because the accuracy of estimates improves with longer datasets.

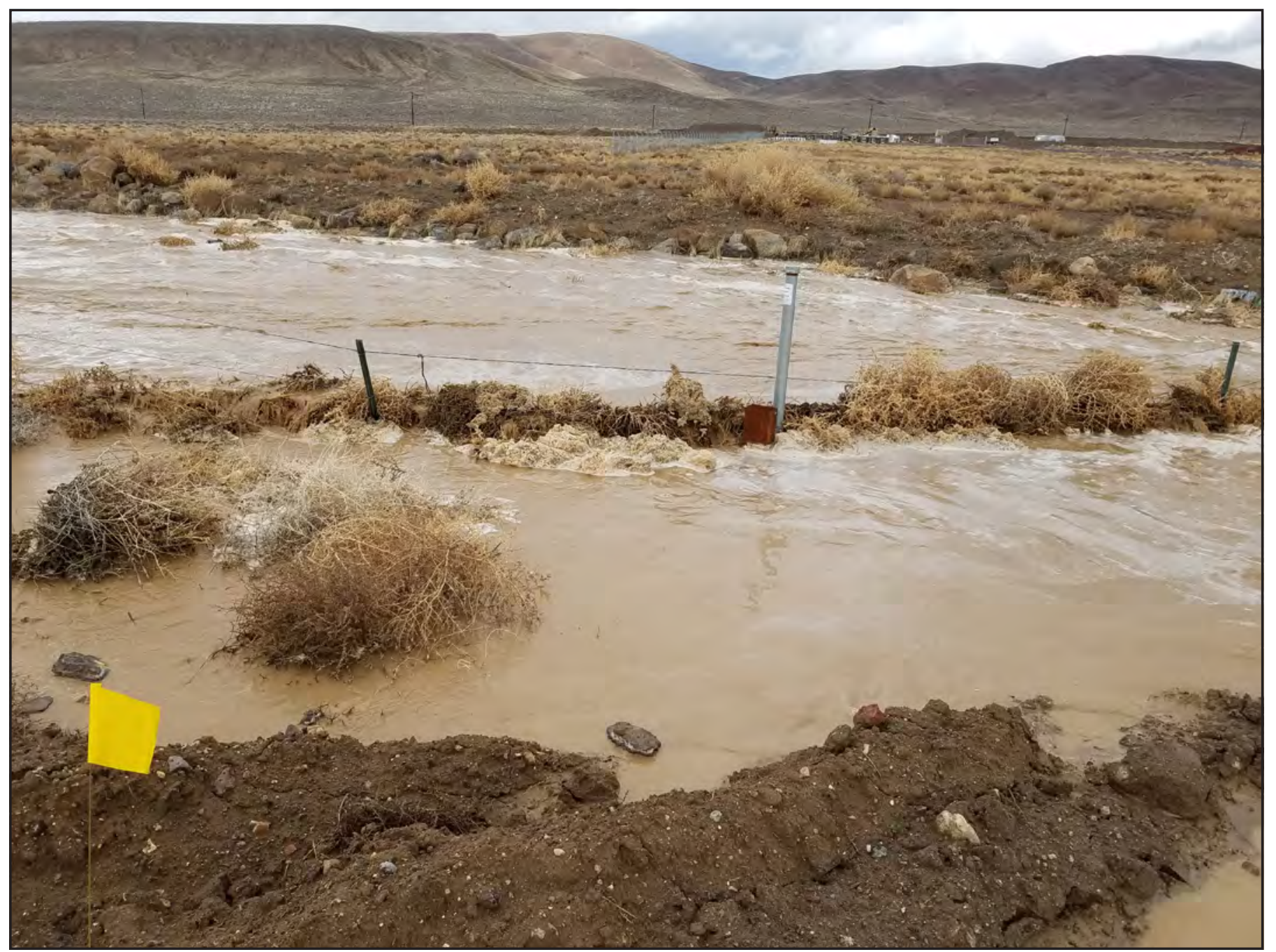

Figure 1. Example of flood event at crest-stage gage at station 10312075 Ramsey Canyon Wash at Hwy 50 at Silver Springs, NV, on February 14, 2019. 


\section{The Program}

Since 1961, the United States Geological Survey (USGS), in a cooperative program with the Nevada Department of Transportation (NDOT), has collected annual peak streamflow data at selected gage sites across the state of Nevada (fig. 2). The purpose of this project has been to characterize annual peak streamflows at the selected gage sites for use in flood frequency analysis. The USGS collects annual peak streamflow data through this project at 26 locations in Nevada. The peak streamflow data are collected through a network of crest-stage gages (CSGs) maintained across the state by the USGS.

A crest-stage gage (fig. 3) is a device that records peak stage between site visits. Crest-stage gages used by the USGS in Nevada typically consist of a 2-inch pipe with vented caps on the top and bottom of the pipe; contained within the pipe is a measuring stick with granulated cork on the bottom. As the flow rises during a flood, the cork contained within the pipe floats on the water surface. As the flow recedes, the cork adheres to the stick and a mark is left on the stick, thereby recording the peak stage of the flood (fig. 3). Lower subsequent peaks also can leave a mark on the stick if enough cork remains in the pipe.

Crest-stage gages are routinely visited every 6 weeks to be inspected, serviced, and for measurements to be obtained at the site using current meters or other methods. Gages also are visited during times of flooding or shortly thereafter to obtain either direct or indirect streamflow measurements. Indirect measurements of streamflow are taken by using surveys of channel geometry, the water surface during the flood peak, and any structures (such as culverts or bridges) to model and estimate the peak streamflow. The peak annual streamflow for gage sites are entered in a publicly available database called the National Water Information System (NWIS), which can be accessed at http://nwis.waterdata.usgs. gov/usa/nwis/peak.

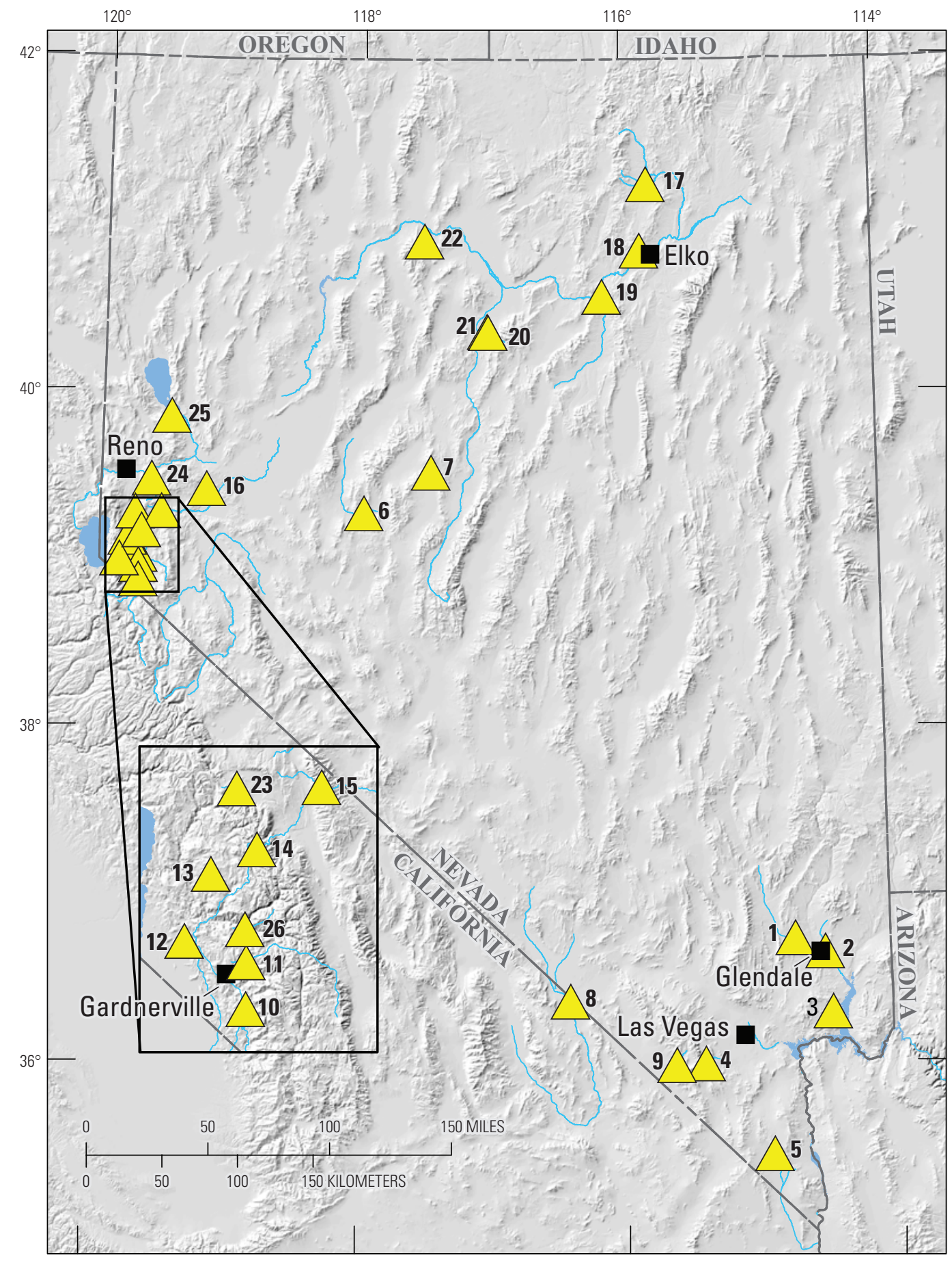

Base from U.S. Geological Survey and U.S. Census Bureau data digital data, 2015

Shaded-relief base from 3DEP 10-meter digital elevation model, 2016.

Universal Transverse Mercator projection, Zone 11

World Geodetic System datum of 1984

Figure 2. Crest-stage gage sites in the network (see "table 1" for the gage-site numbers). 


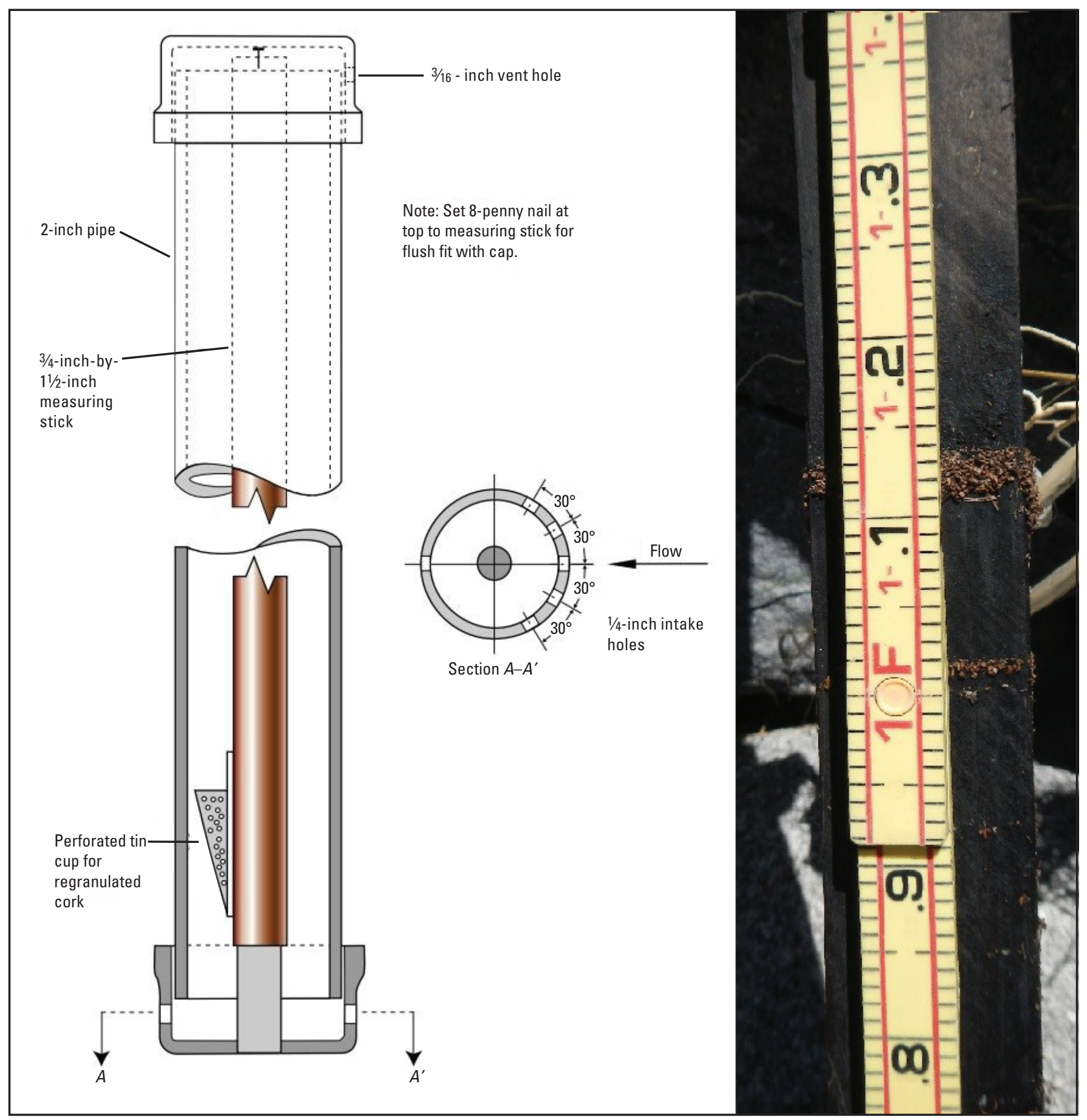

Figure 3. Details of crest-stage gage (CSG) design from Sauer and Turnipseed (2010) and an example of marks left on a CSG stick after multiple peak flows.

\section{Summary}

The annual peak streamflow collected, through this cooperative program with the U.S. Geological Survey (USGS) and the Nevada Department of Transportation (NDOT), at these 26 sites represents 832 peak flow events (table 1; U.S. Geological Survey, 2020). The drainage areas of these sites range in size from 0.62 to 1,542 square miles $\left(\mathrm{mi}^{2}\right)$. The largest recorded peaks ranged from 56 to 12,000 cubic feet per second $\left(\mathrm{ft}^{3} / \mathrm{s}\right)$; these flow events can be used to determine estimates of flood frequency and size. In an effort to minimize flood risk and damage, these estimates of flood frequency and size can be used to design structures to withstand flood damage, designate flood zones, guide management decisions, and direct future planning. 
Table 1. Largest recorded peak streamflow through water year 2020 and associated drainage area at gage sites in Nevada in cooperation with the Nevada Department of Transportation (NDOT).

[ID, identification; $\mathrm{mi}^{2}$, square mile; mm/dd/yyyy, month/day/year; $\mathrm{ft}^{3} / \mathrm{s}$, cubic foot per second]

\begin{tabular}{|c|c|c|c|c|c|c|}
\hline Map ID & Station ID & Name & $\begin{array}{l}\text { Water year } \\
\text { established) }\end{array}$ & $\begin{array}{l}\text { Drainage area } \\
\qquad\left(\mathrm{mi}^{2}\right)\end{array}$ & Date & $\begin{array}{l}\text { Magnitude } \\
\left(\mathrm{ft}^{3} / \mathrm{s}\right)\end{array}$ \\
\hline 1 & 09415851 & Muddy Wash near Moapa, NV & 2012 & 7.8 & $09 / 26 / 2014$ & 2,800 \\
\hline 2 & 09418990 & Weiser Wash near Glendale, NV & 1966 & 43 & $09 / 08 / 2014$ & 12,000 \\
\hline 3 & 09419570 & Echo Wash at Northshore Rd near Echo Bay, NV & 1990 & 106 & 08/15/1990 & 6,000 \\
\hline 4 & 09419680 & Cottonwood Valley near Blue Diamond, NV & 1961 & 18.3 & $01 / 25 / 1969$ & 1,100 \\
\hline 5 & 09423300 & Piute Wash tributary at Searchlight, NV & 1967 & 3.4 & 08/13/1982 & 400 \\
\hline 6 & 10244360 & Dixie Creek Valley Tributary near Eastgate, NV & 1961 & 11 & 08/1961 & 1,480 \\
\hline 7 & 10249417 & Smith Creek Valley Tributary near Austin, NV & 1968 & 0.62 & $07 / 1984$ & 130 \\
\hline 8 & 10251259 & $\begin{array}{l}\text { Amargosa River at Hwy } 127 \text { near California - } \\
\text { Neavada state line }\end{array}$ & 1993 & 1,542 & 09/22/2007 & 660 \\
\hline 9 & 10251980 & Lovell Wash near Blue Diamond, NV & 1965 & 52.8 & $01 / 25 / 1969$ & 4,150 \\
\hline 10 & 10309035 & $\begin{array}{l}\text { Indian Creek above mouth, near } \\
\text { Gardnerville, NV }\end{array}$ & 1986 & 25.4 & 02/19/1986 & 2,100 \\
\hline 11 & 10309075 & $\begin{array}{l}\text { Buckeye Creek at East Valley Road, near } \\
\text { Minden, NV }\end{array}$ & 1992 & 73.8 & 07/14/1992 & 3,000 \\
\hline 12 & 10310410 & Genoa Canyon Creek at Genoa, NV & 1997 & 2.24 & 01/01/1997 & 150 \\
\hline 13 & 10310600 & Voltaire Canyon near Carson City, NV & 1979 & 1 & 01/02/1997 & 118 \\
\hline 14 & 10311450 & Brunswick Canyon near New Empire, NV & 1966 & 12.7 & $01 / 08 / 2017$ & 730 \\
\hline 15 & 10311725 & Six Mile Canyon at Hwy 50 near Dayton, NV & 1986 & 17.29 & $01 / 09 / 2017$ & 663 \\
\hline 16 & 10312075 & $\begin{array}{l}\text { Ramsey Canyon Wash at Hwy } 50 \text { at Silver } \\
\text { Springs, NV }\end{array}$ & 2016 & 44.67 & $01 / 09 / 2017$ & 669 \\
\hline 17 & 10317460 & Gance Creek at Hwy 225 near Tuscarora, NV & 1980 & 20.2 & $04 / 30 / 2006$ & 109 \\
\hline 18 & 10318850 & East Adobe Creek near Elko, NV & 1971 & 6 & 07/27/1971 & 424 \\
\hline 19 & 10322980 & Cole Creek near Palisade, NV & 1962 & 11.4 & $06 / 1983$ & 1,090 \\
\hline 20 & 10326825 & Mill Creek near Battle Mountain, NV & 2006 & Indeterminate & $04 / 15 / 2009$ & 56 \\
\hline 21 & 10326826 & $\begin{array}{l}\text { Mill Creek N. Channel near Battle Mountain, } \\
\text { NV }\end{array}$ & 2011 & Indeterminate & $09 / 02 / 2013$ & 56 \\
\hline 22 & 10328000 & Pole Creek near Golconda, NV & 1961 & 10.7 & 08/05/1961 & 4,000 \\
\hline 23 & 103448600 & Jumbo Wash near New Washoe City, NV & 1986 & 4.86 & 07/22/1986 & 1,230 \\
\hline 24 & 10350100 & Long Valley Creek near Happy Valley, NV & 1956 & 82.6 & $02 / 19 / 1986$ & 5,400 \\
\hline 25 & 10351850 & Pyramid Lake Tributary near Nixon, NV & 1968 & 1.94 & $01 / 08 / 2017$ & 1,220 \\
\hline 26 & 1030909087 & Johnson Wash at Fremont Dr near Minden, NV & 1991 & 10.4 & 07/22/1994 & 1,400 \\
\hline
\end{tabular}

\section{- Kurtiss Schmidt}

\section{References Cited}

Sauer, V.B., and Turnipseed, D.P., 2010, Stage measurement at gaging stations: U.S. Geological Survey Techniques and Methods 3-A7, 45 p., https://pubs.usgs.gov/tm/tm3-a7/.

Sutko, T.E., 2015, Rainfall and flood report September 8, 2014: Clark County Regional Flood Control District, Board of Directors Meeting, Las Vegas, Nev., October 2015, accessed February 5, 2020, at https://gustfront.ccrfcd.org/pdf_arch1/Flood\%20Event $\% 20$ Reports/2014-09-08\%20Rainfall\%20and\%20Flood\%20Event $\% 20$ Report.pdf

Truckee River Flood Management Authority, 2017, Nevada flood awarness: Truckee River Flood Management Authority web page, accessed January 30, 2020, at https://trfma.org/resources/nevadaflood-awareness/.
U.S. Geological Survey, 2020, National Water Information System data, USGS Water Data for the Nation, accessed March 11, 2020, at https://dx.doi.org/10.5066/F7P55KJN.

\section{For more information}

U.S. Geological Survey Nevada Water Science Center 2730 North Deer Run Road Carson City, NV 89701 http://nevada.usgs.gov 